\title{
Author Correction: Closed-loop brain training: the science of neurofeedback
}

Ranganatha Sitaram, Tomas Ros, Luke Stoeckel, Sven Haller, Frank Scharnowski, Jarrod Lewis-Peacock, Nikolaus Weiskopf, Maria Laura Blefari, Mohit Rana, Ethan Oblak, Niels Birbaumer and James Sulzer

Nature Reviews Neuroscience (2017) https://doi.org/10.1038/nrn.2016.164

Published online 22 December 2016

In this article, the affiliation for Mohit Rana was incorrectly listed as the Institute for Biological and Medical Engineering, Department of Psychiatry, and Section of Neuroscience, Pontificia Universidad Católica de Chile, Vicuña Mackenna 4860 Hernán Briones, piso 2, Macul 782-0436, Santiago, Chile. The listed affiliation should have been the following: Departamento de Psiquiatría, Escuela de Medicina, Centro Interdisciplinario de Neurociencias, Pontificia Universidad Católica de Chile, Santiago, Chile; and the Laboratory for Brain-Machine Interfaces and Neuromodulation, Pontificia Universidad Católica de Chile, Santiago, Chile. An acknowledgement to Mohit Rana's funding source was also missing. The following sentence should have been included in the acknowledgments section: M.R. is supported by a Fondecyt postdoctoral fellowship (project no. 3100648).

https://doi.org/10.1038/s41583-019-0161-1 I Published online 25 March 2019 